\title{
Civilisations
}

Revue internationale d'anthropologie et de sciences

humaines

48 | 2001

La question de l'islam et de l'Etat à l'aube du XXIe siècle

\section{L'instrumentalisation de la religion dans les pays musulmans «convertis»*}

\section{Firouzeh Nahavandi}

\section{Q OpenEdition}

1 Journals

Édition électronique

URL : http://journals.openedition.org/civilisations/3462

DOI : 10.4000/civilisations.3462

ISSN : 2032-0442

Éditeur

Institut de sociologie de l'Université Libre de Bruxelles

Édition imprimée

Date de publication : 31 août 2001

Pagination : 85-97

ISSN : 0009-8140

\section{Référence électronique}

Firouzeh Nahavandi, «L'instrumentalisation de la religion dans les pays musulmans «convertis»*», Civilisations [En ligne], 48 | 2001, mis en ligne le 01 août 2004, consulté le 19 avril 2019. URL : http:// journals.openedition.org/civilisations/3462 ; DOI : 10.4000/civilisations.3462 


\title{
L'INSTRUMENTALISATION DE LA RELIGION DANS LES PAYS MUSULMANS «CONVERTIS» ${ }^{1}$
}

\author{
Firouzeh NAHAVANDI
}

\section{INTRODUCTION}

L'étude des rapports entre l'islam et l'État est inépuisable, tant du point de vue théorique que du point de vue empirique. Il y a plus de vingt ans, en Iran, une révolution portait un gouvernement islamique au pouvoir et projetait l'islam révolutionnaire comme idéologie sur la scène internationale. Les idées n'étaient pas neuves, mais l'ouverture du monde et la puissance des médias donnaient à l'événement une publicité non égalée auparavant. Depuis, les événements témoignent que l'islam comme force politique est loin d'être une parenthèse, mais l'a t-il jamais été ?

Dès la fin des années 1970, de nombreux bouleversements dans le monde musulman ont pu donner l'impression, souvent erronée, que la religion a toujours dicté les conduites politiques et imprégné le quotidien des musulmans. Il ne fait évidemment aucun doute que la religion a toujours joué un rôle important dans les pays musulmans et pas seulement dans ceuxci d'ailleurs. Beaucoup de commentateurs, en particulier islamistes, arguent qu'il n'est pas possible de dissocier islam et politique et qu'en conséquence le politique doit se plier au religieux. Sans entrer dans cette discussion qui a été déjà abordée par Olivier Carré (ci avant), le propos essentiel des pages qui suivent sera de montrer comment la religion a non seulement pas nécessairement dirigé le politique mais a pu être instrumentalisée à des fins politiques et étatiques.

Je me limiterai principalement à l'Afghanistan et auxiliairement aux pays d'Asie centrale post-soviétique car il serait difficile d'aborder la question dans son entièreté et de manière exhaustive, même si les conclusions de cette présentation pourraient être, le cas échéant, étendues à d'autres pays.

En effet, les événements récents en Afghanistan, et particulièrement la prise de pouvoir des Taliban $^{2}$ en 1986, ont pu donner l'impression que l'islam dans ce pays a un rôle particulièrement important et imprègne depuis toujours la vie quotidienne et les mœurs. Ce qui est partiellement vrai. Les événements récents ont pu aussi laisser croire que c'est l'islam qui a toujours influencé 
la forme qu'a prise l'État afghan. Or, dans l'histoire afghane ce sont souvent les dirigeants qui ont cherché l'appui de la religion pour légitimer leurs actions ou imposer leur autorité.

De manière identique, si l'on se penche sur l'histoire des pays musulmans de l'ex-URSS, une conclusion similaire s'impose. Tout au long de la domination russe et soviétique, c'est bien l'islam qui a été choisi par des mouvements nationalistes pour contrer l'influence russe. C'est l'islam qui en dépit de divergences idéologiques des protagonistes a été considéré comme l'unique arme capable de réunir les mécontents et de mener à des actions politiques. Ce n'est donc pas pour défendre nécessairement la religion que l'islam a été mis en avant mais à des fins politiques. Après les indépendances, la question se pose différemment comme nous le verrons.

Enfin, plus récemment, le cas de l'expansion de l'islamisme dans le Daguestan et en Tchétchénie illustre aussi l'instrumentalisation de la religion à des fins politiques. Il sera évoqué pour clôturer cette présentation.

\section{CARACTÉRISTIQUES DES SOCIÉTÉS ABORDÉES}

\section{La pluriethnicité}

Toutes les sociétés abordées ci-après sont très diversifiées du point de vue de leur population.

L'Afghanistan est un pays pluriethnique regroupant des peuples d'origines, de langues et de coutumes très diversifiées. Parmi ceux-ci, l'ethnie pashtoune, de langue pashtou, formée d'agriculteurs, d'éleveurs, de commerçants ou encore de nomades; les Tadjiks, persanophones, sédentaires et agriculteurs, les Baloutchs, les Aymaks, les Kazakhs, les Kirkhizs, les Ouzbeks, les Turkmènes, les Hazaras... Depuis la création de l'État afghan, la tradition tribale a été extrêmement importante et particulièrement c'est le pashtounwali (coutumes ancestrales pashtou) qui a influencé les institutions étatiques.

Les pays d'Asie centrale sont aussi une mosaïque de populations diverses. Chacune d'entre elles, outre la population titulaire regroupe en son sein des populations des Républiques voisines, des populations venues du Caucase ainsi qu'un pourcentage important de Russes, résultat de la colonisation. Par exemple, à la veille de l'indépendance, le Kazakhstan était constitué de 36\% de Kazakhs, de 40\% de Russes, de 6,1\% d'Ukrainiens mais aussi de Coréens, de Tatars, d'Ouzbeks ou d'Ouïgours...

L'Ouzbékistan, à la même période, regroupait 68,7\% d'Ouzbeks mais aussi 10,8\% de Russes, 4,2\% de Tatars, 3,9\% de Tadjiks ${ }^{3}$.

Simultanément de nombreux Kazakhs vivent en dehors de leur territoire, entre autres, en Karakalpakie, et de nombreux Ouzbeks au Turkménistan, au Tadjikistan ou encore en Kirghizie.

Dans la République autonome tchétchène de la fédération de Russie, la population se divise en $58 \%$ de Tchétchènes, $18 \%$ d'Ingouches, $16 \%$ de Russes. Le Daghestan présente une variété encore plus grande avec plus de trente ethnies, dont les Avars, $25 \%$, les Darghines, $13 \%$, les Koumyks, $12 \%$, les Lezguiens, $11 \%$, les Russes, $8 \%$, les Tchétchènes, $2,5 \% \ldots$

Face à cette diversité de peuples, de langues, de coutumes et de références, la religion, l'islam en l'occurrence, a toujours été un facteur de cohésion non négligeable. Au siècle dernier, lorsque n'existaient pas, l'Ouzbékistan, le Kazakhstan ou le Tadjikistan comme entité étatique, 
les populations s'identifiaient aux vieilles segmentations ethniques ou tribales ou à l'umma, la communauté des croyants en islam. Comme en Afghanistan, l'islam a été l'unique trait d'union de la mosaïque ethnique.

\section{Une population musulmane}

L'ensemble des territoires composant l'Afghanistan actuel ne seront islamisés qu'à la fin du IX' siècle, le Nouristan au XIX ${ }^{e}$ siècle.

Aujourd'hui, la grande majorité de la population afghane est musulmane. Elle est à $85 \%$ sunnite de rite hanéfite, le reste étant shiite ou ismaélite. «Dans un pays où la référence à la nation est très récente, où l'État est perçu comme extérieur à la société et où l'allégeance va au groupe communautaire, l'islam reste la seule référence commune à tous les Afghans» ${ }^{4}$. Cependant, comme le démontre Olivier Roy, l'ancrage sociologique de la religion est très différent selon les milieux, de même que les références idéologiques à la religion varient selon les groupes.

Il faut, en conséquence faire une distinction entre la religiosité populaire, l'orthodoxie scolastique et juridique des uléma, la mystique des soufis et l'islam politique des islamistes ${ }^{5}$.

La religion populaire structure le quotidien et institutionnalise un rapport au sacré assez différent de celui qui peut se déduire de la théologie officielle. C'est l'islam tel qu'il est vécu au village. La religion structure l'espace et le temps. Espace du village, centré autour de la mosquée, mais aussi espace monde avec ses cercles concentriques, de l'umma, des autres religions et des athées. Rythme du jour avec ses cinq prières, rythme de la langue avec les adresses, les formules de politesse et les interjections qui mentionnent le nom de Dieu.

Au centre de cela, le mollah qui appartient au village et non au clergé et qui n'a souvent pas de relations avec le clergé supérieur. En zone tribale, le mollah est l'employé du village, c'est l'artisan des rites uniquement.

L'islam fournit donc une morale sociale mais est aussi une forme de spiritualité qui s'inscrit dans les comportements et qui donne une transcendance, un espace de méditation personnelle un accès à l'universel au delà de l'immanence des règles de la vie communautaire.

La mystique soufie incarnée par trois ordres, nakhshbandi, qaderiya et tchechtiyya touche surtout les classes moyennes des villes et des bourgs. Approche qui privilégie la révélation ésotérique plus que la révélation exotérique.

Enfin, il faut évoquer l'islam des uléma, groupe instruit qui se définit comme corps par sa formation plus que par sa place dans l'institution politique. Les uléma sont des lettrés et non des intellectuels. Formés selon un cursus millénaire commun à tout le monde musulman, ils ont plus le sentiment d'appartenir à la communauté musulmane qu'à une nation particulière. Les uléma en tant que tel n'ont pas vocation au pouvoir quoiqu'ils aient le sentiment d'incarner la légitimité islamique. Ils ont pour fonction de vérifier la conformité ou plutôt la non contradiction de la politique avec la sharia (loi musulmane). Leur objet est la société civile, non pas l'État, donc pas le politique en tant que tel.

Les islamistes, quant à eux, incarnent l'irruption d'une référence islamique dans les domaines politiques, sociaux et économiques. Ils posent l'islam en idéologie politique apte à concurrencer les grandes idéologies du $\mathrm{XX}^{\mathrm{e}}$ siècle. 
L'islam arrive en Asie centrale avec la conquête arabe au VIII ${ }^{\mathrm{e}}$ siècle et s'étend peu à peu jusqu'au $\mathrm{X}^{\mathrm{e}}$ siècle. Toutefois, son influence sera différente dans les steppes et les montagnes par rapport aux régions urbaines et politiquement très organisées. C'est dans ces dernières qu'il s'est particulièrement bien ancré, les sédentaires d'Asie centrale ont probablement toujours eu un sentiment d'appartenance plus prononcé à l' umma, le résultat étant encore visible aujourd'hui en Ouzbékistan. Néanmoins, les croyances musulmanes font partie du quotidien des sociétés. La conscience musulmane est présente partout et les références à Allah dans le discours sont fréquentes. Même si les peuples d'Asie centrale sont «convertis», que l'athéisme de la période soviétique a laissé des traces, la religion demeure un point commun important et a constamment été utilisé comme élément de l'identité par rapport à l'envahisseur russe.

\section{Un État récent}

Stricto sensu, l'Afghanistan n'existe en tant qu'État que depuis 1880, date à laquelle les Anglais reconnaissent l'Émir Abdur Rahman comme roi d'Afghanistan. Toutefois, pour beaucoup, l'État afghan naquit en 1747, lorsque Ahmad Khan Abdali se fit élire Shah et adopta le titre de Durrani. Cet événement est considéré comme l'acte de naissance officiel de l'Afghanistan et Ahmad Shah comme père de l'Afghanistan. L'empire du roi n'était pourtant pas un État au sens moderne du terme (voir plus loin).

Quant aux États actuels de l'A Asie centrale post-soviétique, jusqu'à la colonisation des tsars, ils formaient des entités à part entière, comme entre autres, l'Émirat de Bukkhara, les Khanates de Khiva ou de Khokand. En 1865, les Russes font de cette région une entité administrative, le Turkestan. C'est sous Staline, que le territoire sera divisé en cinq entités, Kirghizie, Kazakhstan, Ouzbékistan, Turkménistan et Tadjikistan. Cette division est faite sur la base de la nationalité telle que définie par Staline : communauté humaine stable, historiquement constituée, fondée sur la langue, le territoire, la vie économique et spirituelle. Elle répondait aussi au besoin de casser l'éventuelle solidarité qui aurait pu se constituer entre des peuples partageant une même religion, et ayant des références communes et de là faciliter l'expansion du communisme dans une région qui comptait très peu de partisans avant la révolution. Toutefois, théoriquement, ces États faisaient partie d'un ensemble fédéral, association librement consentie d'États ayant droit à la sécession, aux représentations diplomatiques, à l'activité parlementaire, au niveau de la république et au niveau fédéral. Néanmoins, dans la pratique rien n'était vraiment respecté.

Quoi qu'il en soit, ce n'est qu'en 1991, que cinq États indépendants au sens moderne verront le jour.

\section{LES INSTRUMENTALISATIONS DE LA RELIGION EN AFGHANISTAN}

L'essentiel de mon propos dans cet article est de montrer qu'en raison des caractéristiques spécifiques de l'Afghanistan, et en particulier son caractère tribal, l'islam a été constamment instrumentalisé mais toujours de manière différente et par des acteurs différents. Parmi ceuxci les rois, les tribus, la résistance et les pays étrangers.

\section{L'instrumentalisation de la religion par les rois}

L'empire de Ahmad Shah est assez conforme au modèle de conquête tribale de Ibn Khaldoun. Il tient autour de la personnalité charismatique du roi. Trois-quart de ses revenus proviennent des conquêtes, et particulièrement de l'Inde ${ }^{6}$. Ainsi, comme les ressources sont 
extérieures, Ahmad Shah n'a pas à se préoccuper de taxer les tribus desquels dépend son pouvoir. Les leaders tribaux s'occupent de l'organisation militaire (armée asiatique).

Néanmoins, le roi essaie de créer un pouvoir militaire indépendant et essaie de restructurer les tribus dans son intérêt, par exemple en nommant des chefs, en engageant des non pashtouns et surtout en utilisant l' islam pour légitimer le pouvoir central. Il imposera la sharia, surtout dans les villes. En effet, face à l'irrédentisme des tribus pashtouns, face à leur aversion à se plier à une autorité centrale et à laisser contrôler leurs affaires internes, les rois afghans ont dû constamment s'appuyer sur la légitimité islamique. Ceci restera vrai jusque dans les années 1960 où la notion d'État-nation commencera à faire son chemin.

Une illustration de ce besoin vient du roi Abdur Rahman qui régna de 1880 à 1901 et d'un firman (une proclamation) qu'il nous reste, reproduite par Lord Curzon, et que le roi avait fait écrire à l'intention de ses sujets' ${ }^{7}$. Dans ce récit, le roi fait constamment appel à la légitimité divine.

L'un des principes de la royauté musulmane, dans sa version afghane, entre autres, est l'idée de Dieu comme créateur de l'univers et ultime juge des affaires humaines. Tout pouvoir émane de Dieu et c'est lui qui détermine la part que chacun reçoit, à savoir la position que l'on a à sa naissance et celle que l'on va atteindre. La proclamation de Abdur Rahman se base sur la notion de contrôle divin en alignant constamment les intérêts du monarque sur ceux de Dieu. Le roi, comme les autres êtres humains, est soumis à l'autorité divine, dit-il, il sera également jugé pour ses actions et son obéissance mais le roi a aussi mission de garantir que ses sujets puissent remplir leurs devoirs de musulmans. La biographie du roi est ainsi truffée d'épisodes sur l'injustice de certains monarques qui ne remplissent par leur fonction divine.

Abdur Rahman fait aussi référence à son élection divine par référence à une série de miracles qui précèdent sa prise de pouvoir et à une multitude de rêves divinement inspirés.

Comme beaucoup de dirigeants musulmans avant lui et après lui, Abdur Rahman utilise les rêves non seulement pour légitimer ses actions mais aussi pour leur donner un caractère inévitable.

La notion de gratitude, qualité requise chez un bon croyant est aussi utilisée dans le récit précité en étendant cette relation aux sujets par rapport à leur roi. Ainsi les personnes mécontentes témoigneront de l'ingratitude non seulement au roi mais à Dieu. Il semble à cet égard que le roi ait été conscient du danger que comportait l'ethos tribal de compétition, entre autres, pour son armée. Il était conscient du décalage entre la notion de suprématie telle que véhiculée dans les tribus et la notion de hiérarchie et d'obéissance telle que nécessaire dans l'armée.

Abdur Rahman présente la désobéissance à l'État et la convoitise de la position de ses supérieurs comme des illustrations d'ingratitudes passibles de jugement divin.

En résumé, le fait que le roi sente le besoin de soumettre son peuple à ses remarques démontre le danger qu'il ressent par rapport au menaces tribales de son autorité. Il utilisera constamment l'autorité religieuse pour ses actions politiques comme par exemple pacifier l'opposition au Nord du pays ou étendre son autorité à l'Est et au Centre. Il ira même jusqu'à déclarer qu'il est le seul à pouvoir déclarer la jihâd. Toutefois ce que l'Émir de fer pouvait se permettre par sa puissance ne pouvait se transmettre à ses descendants. 
L'Émir a ainsi, pour limiter le tribalisme, mis en avant la légitimité islamique. Il est Émir de droit divin, défenseur de la religion et imposera la sharia pour mieux assurer un droit étatique. Il essaiera aussi d'intégrer les uléma dans l'appareil d'État (par le contrôle des madrasas,des waghfs...). En définitive, au XIX ${ }^{\mathrm{e}}$ siècle, la monarchie afghane voyait en la sharia un instrument efficace et commode pour cimenter la société en une unité gouvernable.

En outre, jusqu'en 1924 (date de l'abolition du khalifat ottoman et des premières vagues de modernisation du roi Amanullah), les rois légitimeront l'État au nom de la défense de l'islam face aux infidèles britanniques et russes et face aux hérétiques persans.

La référence à l' umma joue un rôle important en Afghanistan, mais jusqu' au début du XX $\mathrm{X}^{\mathrm{e}}$ siècle, le panislamisme n'est pas une doctrine politique, c'est une référence idéologique qui accompagne une réaction à l'égard d'une intrusion infidèle contre la communauté musulmane. Les premiers à penser le panislamisme en termes politiques sont les souverains afghans. Pour eux, Amanullah compris, le panislamisme est un moyen de légitimation de l'État naissant et un facteur de politique étrangère qui permet d'accroître le rôle de l'Afghanistan dans la région, tout en évitant de se laisser entraîner dans une politique de déstabilisation de l'Inde britannique ou du Turkestan soviétique. Toutefois, si, le cas échéant, le panislamisme met en danger l'État afghan, les rois choisissent la prudence contre les pressions des uléma en faveur d'une intervention. Citons à titre d'exemple, le cas de la mutinerie indienne de 1847 , ou encore le refus de soutenir les basmatchis d'Asie centrale par Amanullah.

\section{L'instrumentalisation de la religion par les tribus}

Traditionnellement, l'Émir s'appuyait sur le consensus de la communauté (docteurs de la loi et croyants) de telle sorte qu'il était à la fois l'autorité suprême dans les domaines religieux, politique, administratif et judiciaire. Il tenait compte également des apports coutumiers. Pour les institutions étatiques ce sont les principes coutumiers de l'ethnie pashtoune, ou pashtounwali qui prévalaient.

Cet ordre traditionnel sera bouleversé par la prise de pouvoir du roi Amanullah, en 1919. Celui-ci se lance dans la transformation des structures sociales et politiques pour faire de l'Afghanistan un État-nation moderne. Les réformes incluent des mesures dans trois domaines primordiaux pour le contrôle social, les taxes, la propriété et le transport.

Exigence de taxes en liquide, simplification des droits de douanes et contrôle des droits de douane, abolition de l'esclavage...

Amanullah met aussi fin aux allocations payées aux tribus. D'un point de vue social, il transforme l'enseignement, interdit le voile....

Cet essai de modernisation conservatrice, pour employer l'expression de Bertrand $\mathrm{Badie}^{8}$, va entraîner le mécontentement des tribus et le roi devra faire face aux révoltes tribales contre la centralisation administrative, interprétée comme un empiétement inconcevable sur leurs affaires et une intrusion dans des sphères qui lui étaient hostiles. La politique de modernisation d'Amanullah lui aliène le clergé et les chefs de tribus.

C'est à cette période qu'un désaccord fondamental va s'introduire sur le rapport entre défense de l'islam et occidentalisation. Les uléma estiment que la défense de l'islam passe par la réislamisation de la société, par le retour à la sharia, alors que pour le roi la défense de l'islam est un thème politique anti-impérialiste et passe par l'occidentalisation. 
Les révoltes successives seront bientôt menées par Bacheyé saqaf, tadjik aux origines douteuses, qui prendra la tête du mouvement au nom de la défense de l'islam. Pour O. Roy c'est le candidat de la coalition fondamentaliste qui a sapé le pouvoir d'Amanullah.

Bacheyé saqaf sera couronné roi mais son règne sera de courte durée puisque le pouvoir sera très vite repris par une confédération tribale qui nommera Nadir khan comme roi par la grande jirga et sans se référer aux uléma.

De manière générale, en Afghanistan, le pouvoir des rois a été inversement proportionnel à celui des tribus et des uléma. Nadir Khan, successeur de Bachéyé saqaf, s'étant appuyé sur les uléma, le pouvoir deviendra fortement tributaire de ceux-ci.

\section{Le rôle de la guerre et l'instrumentalisation de la religion}

Dans la mosaïque ethnique et religieuse qu'a constitué le territoire de l' actuel Afghanistan, l'islam a toujours été particulièrement méfiant par rapport aux influences étrangères et a constitué historiquement un point de ralliement de beaucoup de mouvements de résistance. En particulier, il faut citer celui contre les Britanniques, lancé par Sayyid Ahmed Barelvi (17861831) qui, ignorant les leaders tribaux et se reposant sur les uléma, annonça la jihâd contre les Britanniques. Barelvi demandait que la sharia remplace les lois tribales.

La prise de pouvoir des communistes, puis l'invasion soviétique ont de même provoqué une politisation de la société afghane et magnifié le rôle de l'islam comme force politique, rôle que dans d'autres circonstances, il n'aurait probablement pas eu. Tous les récits s'accordent pour présenter l'islam afghan comme traditionnellement modéré.

Toutefois, par exemple, dans les camps de réfugiés, les éléments les plus radicaux des groupes islamistes profitèrent de l'opportunité pour endoctriner la population tout en alphabétisant.

En outre, la guerre et l'instabilité politique, la succession de régimes et de pouvoir, le manque de continuité en un mot, ont renforcé le rôle vital de l'islam et de la sharia comme facteur stabilisant.

Il est tout à fait significatif que les différents protagonistes dans la guerre civile aient également utilisé l'islam par l'intermédiaire de l'appel à la jihâd. Ainsi, les moudjahidin ont d'une part fait appel à la jihâd et à la résistance et d'autre part le gouvernement communiste, tirant les leçons de ce succès, sortira de ses rouages un mollah déclarant la jihâd à la résistance présentée comme étant l'instrument des USA et d'Israël ...Le pouvoir communiste espérait amadouer le clergé traditionnel en changeant le drapeau du rouge vers sa couleur originale, vert, noir et rouge, ou encore en modifiant le nom du parti qui devint vatan (patrie) au lieu de PDPA (parti démocratique du peuple afghan). Ces tentatives ne seront pas couronnées de succès et le régime de Kaboul fut exclu de l'Organisation de la Conférence islamique.

La guerre civile et la destruction de la société civile ont donné momentanément une rôle à la sharia là où il y a avait des personnes qualifiées à l'exercer. Toutefois, la guerre civile a également démontré l'extrême rivalité ethnique dans ce pays et surtout l'incapacité de l'islam à surmonter et contrôler ces rivalités. Encore aujourd'hui les loyautés et les identités ethniques demeurent importantes en dépit de l'existence d'une population musulmane homogène.

Il est évident que parmi les facteurs ayant contribué à la formation de la résistance et à l'exode de plus de cinq millions de réfugiés, la foi en l'islam a été très importante. 
Comparativement, le nationalisme afghan ne bénéficie pas d'une grande base sociale. Ainsi, l'adhésion à une vison non séculariste de l'islam a aidé les modjahidin. Pourtant, sans l'existence d'un ennemi extérieur (les troupes soviétiques) ou intérieur (un régime athée), le semblant de front uni des modjahidin s'est transformé en rivalité ethnique.

\section{L'instrumentalisation de la religion par les pays étrangers}

Les Taliban, littéralement étudiants en religion, qui ont pris Kaboul en Septembre 1996, ne représentent qu'une des manifestations de la problématique afghane, mais également leur prise de pouvoir est l'occasion de nombreux questionnements sur l'islam comme religion ou comme idéologie politique et sur les relations politiques et économiques internationales.

Leur apparition sur la scène politico-militaire comme mouvement armé ne tombe pas du ciel. Elle prend ses sources à l'évacuation de l'armée rouge dès 1989. En effet, on n'assistera pas à une victoire rapide de la résistance mais à une nouvelle guerre civile. Antérieurement unies contre l'ennemi soviétique, les différentes factions afghanes, auxquelles participent isolément des Taliban, s'entre-déchirent ${ }^{9}$.

La population afghane est exténuée, elle vit dans l'insécurité et l'instabilité et accueillera somme toute les victoires des Taliban comme une libération (la légende veut que le mollah Omar ait reçu un appel de dieu, lui enjoignant de mettre fin aux combats fratricides dévastant l'Afghanistan! (cf. Abdur rahman...).

Toutefois, ce qui est décisif dans la victoire de ces étudiants en religion est le soutien pakistanais. En effet durant la guerre, le Pakistan a soutenu toutes les factions de la résistance, et en cela a été financé et encouragé par les USA, l'Arabie Saoudite et beaucoup d'autres pays du Golfe. Mais, lorsque pendant la guerre du Golfe, les principales factions soutiennent Saddam Hussein et aussi parce que les Pakistanais se rendent compte que leur principal candidat Hekmatyar n'arrive pas à contrôler l'Afghanistan, ils tourneront leur soutien vers les Taliban.

Cette option présente de nombreux avantages. Elle assure aux Pakistanais une profondeur stratégique face à l'Inde. Depuis bien longtemps, l'idée d'un Afghanistan faible, inféodé dans le meilleur des cas a fait son chemin au Pakistan. D'un point de vue intérieur, le soutien de l'option islamiste conforte les propriétaires terriens inquiets de toute option socialiste. D'un point de vue extérieur, le Pakistan s'appuie sur l'Arabie Saoudite dont l'idéologie est proche de sa pensée islamiste, qui finance la résistance et reçoit de la main d'œuvre bon marché du Pakistan.

Mais aussi, les Taliban garantissent au yeux des Saoudiens, l'endiguement de toute avancée shiite.

Enfin, les Taliban garantissent aux compagnies pétrolières américaines et saoudiennes un accès direct aux nouveaux États d'Asie centrale, riches en hydrocarbures et prometteurs d'importants marchés. Comme l'a écrit $O$. Roy «les USA reprennent en Afghanistan la recette de l'Aramco (compagnie pétrolière américaine) dans l'Arabie Saoudite des années 30 : fondamentalisme islamique, tribus et pétrole» ${ }^{10}$.

La CIA et les services de sécurité d'Unocal (compagnie pétrolière saoudienne) ont fourni des armes et des instructeurs militaires à plusieurs milices qui combattent en l'occurrence autant pour Allah que pour les dollars. 
L'INSTRUMENTALISATION DE LA RELIGION PAR LES MOUVEMENTS NATIONALISTES : LE CAS DE L'ASIE CENTRALE

Dès la conquête russe, dans une région où la majorité de la population fait référence à des identifications supranationales comme l'islam ou sub-nationales comme le clan, les élites centre-asiatiques ont très vite interprété la défaite face aux Russes comme le déclin de leur communauté religieuse. C'est autour de l'islam qu'ont tourné les questions de l'occupation. Car il sera clairement perçu par tous comme le dénominateur commun des populations conquises depuis 1552 par les Russes et comme le seul moyen d'action possible.

Les réformistes et les nationalistes s'engagent très tôt dans un processus de recherche d'identité qui permette d'unifier la communauté musulmane vivant dans l'empire des tsars. Il s'agit toutefois d'un panislamisme doublé de pan-turquisme, dans la mesure où une partie de la population est turcophone et que le khalife ottoman est turc.

C'est dans ce cadre qu'il faut comprendre les mouvements qadims (anciens) et jadids (nouveaux) à la fin du XIX ${ }^{\mathrm{e}}$ siècle. Les premiers préconisent la stricte application de la sharia. Ils veulent un changement établi dans le cadre des traditions musulmanes ${ }^{11}$. Leur audience sera importante car dans leurs rangs se trouvent beaucoup de clercs et de soufis ${ }^{12}$. Les jadids, nationalistes issus aussi des écoles coraniques s'orientent vers un panislamisme teinté de panturquisme. Ils sont influencés par le mouvement intellectuel qui, dans le monde musulman, s'élève contre l'impérialisme. En particulier, par les idées de Mahmoud Tarzi qui publie dans les années 1920 le journal libéral Siraj-ul-Akbar à Kaboul. Cette publication est l'instrument de propagande de deux thèmes, le colonialisme et l'impérialisme doivent disparaître. Elle est l'expression d'un lien entre islam, modernisation et nationalisme.

Le mouvement basmatchi expression d'une opposition nationaliste qui voit le jour dès 1917 s'inspire aussi de l'islam. Toutefois, face aux contre-attaques soviétiques, menées sous les formes militaire, sociale et diplomatico-militaire, il s'essoufflera et son dernier dirigeant, Enver Pacha, ne réussira pas à maintenir l'élan nationaliste, car c'est la haine de l'infidèle et la défense de l'islam qui animent les guerriers et non la conscience nationale.

Bien plus tard, dans la deuxième moitié du XX' siècle, ce sera encore l'islam qui sera utilisé par les mouvements d'opposition et ce même dans des pays où l'islam a toujours été moins visible. En effet, au Kazakhstan par exemple où le débat sur le fait que l'islam devrait s'adapter aux coutumes kazakh est très présent, les intellectuels, pour préserver leur identité culturelle, en viennent à considérer qu'il faut tenir compte de la religion dans l'histoire et la culture. Car, c'est bien l'islam qui distingue les Russes des Kazakhs et comme facteur distinctif, il est progressivement devenu, pour les intellectuels séculiers, comme il l'était pour la hiérarchie musulmane, un élément de la culture kazakh.

En Ouzbékistan, là où la tradition musulmane est plus ancrée, dans les années 1960, le premier secrétaire du parti communiste inaugure une période de glorification de la nation ouzbek et d'affirmation de la spécificité de l'islam. Dans cette république, les campagnes anticorruption qui affectent les cadres, dans les années 1980, au lieu de redonner confiance à la population, heurte le sentiment national et c'est autour de la religion que se cristallisent les mécontentements. La campagne athéiste de Gorbatchev n' arrangera en rien les choses. Nombre de cadres seront évincés et ils se tournent aussi vers l'islam. Karimov, premier secrétaire du parti et futur président récupère les élans nationalistes à son profit. Il amorce entre autres, un rapprochement avec les mosquées et forge une alliance avec le mufti Muhammad Yusuf du directorat spirituel musulman de Tashkent. 
Ainsi, l'opposition à la domination et à la présence russe en Asie centrale sont passées par une instrumentalisation de la religion qui étant perçue comme point commun des populations très diversifiées et signe distinctif par rapport aux populations slaves, est apparue comme un instrument de lutte et de prise de conscience à des mouvements d'opposition même laïques.

A la suite des indépendances, la recherche de l'identité pousse les États à des incursions dans la sphère de la religion, néanmoins tous les dirigeants ont proclamé le caractère lä̈que de l'État. En outre, la montée des tendances islamistes et les troubles chez les voisins, comme l'Afghanistan, ou encore au Tadjikistan, ont incité les dirigeants à beaucoup de prudence et surtout a mettre en place un islam officiel. Dans ce sens, en Ouzbékistan, une université islamique a été créée en avril 1999. Il faut toutefois constater que face à cela, les mouvements extrémistes s'activent en dehors de tout contrôle étatique.

A l'ère postcommuniste, l'islam radical attire tous les désespérés et les laissés pour compte de l'ordre libéral qui y cherchent une nouvelle raison d'espérer. En particulier, dans la vallée de Ferghana, où traditionnellement l'islam avait un ancrage plus grand et où la baisse du niveau de vie est considérable, les jeunes se réfugient derrière une idéologie nourrie par les Wahhabites ${ }^{13}$ et dans des mouvements financés par l'étranger dans lesquelles se retrouvent pèle mêle des activistes ouzbeks, tadjiks ou encore afghans...

\section{CONCLUSION : LA MONTÉE DE L'ISLAMISME DANS LE CAUCASE NORD}

Sous l'URSS, dans l'élaboration des contours du fédéralisme, distinction avait été faite entre les régions non russes situées à l'intérieur et celles de la périphérie. Le statut de région autonome et de république était donné aux premiers et celui de République de l'union ou République socialiste aux seconds. La différence entre les deux résidait théoriquement dans le fait que les secondes, au contraire des premières, étaient reconnues comme ayant droit à la sécession ${ }^{14}$. La constitution de la Russie n'envisage pas ce cas. Ainsi, la Tchétchénie fait partie intégrante de son territoire et les velléités d'indépendance de cette république ou du Daguestan voisin feront inévitablement réagir Moscou.

Le Daguestan était très laïcisé et ses habitants pratiquent depuis toujours un islam modéré. La Tchétchénie n'a pas non plus été le lieu par excellence d'une rivalité entre le christianisme et l'islam. Dans ce cadre, la compréhension des conflits qui secouent le Caucase Nord sont à rechercher ailleurs. En particulier, il faut considérer les richesses pétrolières qui poussèrent, en 1991, le président tchétchène Doudaïev, à proclamerl'indépendance, dans le but d'en récupérer les revenus. Dans la guerre qui opposa de 1994 à 1996, les Tchétchènes aux Russes, les premiers qui ne s'étaient pas auparavant illustrés par un militantisme religieux, trouvèrent dans l'islam à la fois l'étendard d'une jihâd antirusse et un moyen d'obtenir des crédits auprès des mouvements islamistes et des pays qui les soutenaient ${ }^{15}$.

La mort de Doudaïev, en 1996, divisa ses partisans. D'un côté, la «branche historique» voulant poursuivre la lutte et de l'autre une faction plus inquiète des répercussions de la guerre sur les rendements économiques et qui parviendra à un accord avec Moscou : aide financière russe, partage des bénéfices pétroliers et promesse de référendum au bout de cinq ans. Cette évolution poussa la faction historique des combattants tchétchènes à manifester son opposition par un ancrage dans le radicalisme. «De prétexte, l'islam se transforma en but» ${ }^{16}$. Les changements de position de Moscou, notamment la décision de mettre fin aux subsides et de trouver des remplacements aux oléoducs qui traversent la Tchéchénie, renforceront la position des islamistes, dont Chamil Bassä̈ev. En 1998, ce dernier lance l'idée de la réunification du 
Daguestan et de la Tchétchénie, qui s'illustrera par ailleurs par la proclamation, dans les montagnes du sud du Daguestan, de la fusion de trois villages se réclamant du wahhabisme en une république islamique indépendante.

Outre les convoitises tournant autour de l'enjeu pétrolier, la situation catastrophique de l'économie de ces deux républiques favorise la cristallisation des mécontentements dans une opposition islamiste. L'agriculture du Daguestan est dans état déplorable, $30 \%$ des habitants serait au chômage ( $80 \%$ selon certaines sources) ${ }^{17}$. Cette république, comme la Tchétchénie, souffre de la crise économique provoquée par la transition et de la corruption endémique qui sévit comme partout ailleurs dans l'ex-URSS. L'aspiration des populations à de meilleures conditions de vie poussent une partie de ceux-ci vers le radicalisme religieux. Aux élections de 1993, 63,3\% des voix se sont portés sur le candidat islamiste. Déçue par l'ère post-soviétique, de nombreux Daguestanais sont favorables à un État islamique. Néanmoins, la politisation de l'islam n'a pas encore abouti à la proclamation, comme en Tchétchénie, d'un État islamique et à la réintroduction de la sharia probablement d'ailleurs en raison de l'extrême division ethnique.

Toutefois, nul ne peut aujourd' hui prédire les conséquences à cours, moyen et long terme des bombardements et de la guerre sur la vision des populations du Caucase Nord à propos des «infidèles qui ont massacré les populations civiles».

\section{BIBLIOGRAPHIE}

Afghanistan, La colonisation impossible, 1984, Paris, Cerf.

AKBAR, A. et HART, D. M., eds, 1984, Islam in Tribal Societies : From the Atlas to the Indus, London, Routledge and Kegan Paul.

ANDERSON, J., 1997, The International Politics of Central Asia, Manchester New York, Manchester University Press.

ARNEY, G., 1990, Afghanistan, London, Mandarin.

BADIE, B., 1986, Les deux États, Paris, Fayard.

BARBER, B.R., 1995, Jihad vs McWorld, How Globalism and Tribalism are Reshaping The World, USA, Ballantine Books Edition.

BARRY, M., 1989, La résistance afghane, du grand moghol à l'invasion soviétique, Paris, Flammarion.

BRILL OLCOTT, M., 1996, Central Asia's New States, Washington DC United States Institute of Peace.

CAGNAT, R. et Jan, M. , 1990, Le milieu des empires, Paris, Robert Laffont.

CARATINI, R., 1992, Dictionnaire des nationalités et des minorités de l'ex-URSS, Paris, Larousse.

CRITHLOW, J., 1991, Nationalism in Uzbekistan, Boulder, Westview Press.

DUPRÉE, L., 1980, Afghanistan, Princeton, Princeton University Press.

EDWARDS, D. B., 1996, Heroes of The Age, Los Angeles, University of California Press. 
GLEASON, G., 1997, The Central Asian States Discovering Independance, Boulder, Westview Press.

GOLDENBERG, S., 1994, Pride of Small Nations, The Caucasus and Post-Soviet Disorder, London and New Jersey, Zed Books.

GREGORIAN, V., 1969, The Emergence of Modern Afghanistan : Politics of reform and Modernization, 1880-1946, Stanford, Stanford University Press.

HAGHAYEGHI, M., 1995, Islam and Politics in Central Asia, London MacMillan.

HALLIDAY, Fr., 1995, Islam and the Myth of Confrontation, London, Tauris.

HIRO, D., 1988, Islamic Fundamentalism, London, Paladin.

HIRO, D., 1995, Between Marx and Muhammad, The Changing Face of Central Asia, London, Harpers Collins Publishers.

MAGNUS, R. H. and NABY, E., 1997, Afghanistan, Mullah, Marx and Mujahid, Boulder, Wrstview Press.

MARSDEN P., 1998, The Taliban, South Asia, Oxford University Press.

NAIPAUL, V.S, 1998, Beyond Belief, Islamic Excursions Among The Converted Peoples, London, Abacus.

POULLADA, L., 1973, Reform and Rebellion in Afghanistan, 1919-1929, Ithaca, Cornell University Press.

RASHID, A., 1994, The Resurgence of Central Asia : Islam or Nationalism, London, Zed Books.

ROY, O., 1985, L'Afghanistan, islam et modernité politique, Paris, Seuil.

RUBIN, B., 1995, The Fragmentation of Afghanistan, New Haven and London, Yale University Press.

RUBIN, B.R., 1995, The Fragmentation of Afghanistan, USA, Yale University Press.

\section{NOTES}

1 L'expression converti est reprise à l'écrivain V.S. Naipaul, dans son livre Beyond Belief, Islamic excursions among the Converted Peoples, London, Abacus, 1998. L'auteur emploie le terme pour tous les pays musulmans non arabe. Nous avons nous même à maintes reprises fait cette différence en employant le terme pays musulmans non arabe dans nos écrits précédents.

2 Littéralement, étudiants d'écoles islamiques.

3 Les pourcentages sont repris à Roger Cartini, Dictionnaire des nationalités et des minorités de l'exURRS, Paris, Larousse, 1992. Ces pourcentages ont aujourd'hui subi de grands changements, en particulier, le nombre de Russes dans chacune des républiques a diminué de manière significative, néanmoins, ces pays demeurent multiethnique.

4 O.Roy, «L'islam en Afghanistan», in Afghanistan, la colonisation impossible, Paris, Les éditions du Cerf, 1984, pp. 81-117, p. 81.

5 Voir pour cette distinction Olivier Roy «L'islam en Afghanistan» in Afghanistan, la colonisation impossible, Paris, les éditions du Cerf, 1984, pp. 81-117. 
6 Voir, B. Rubin, The Fragmentation of Afghanistan, State Formation and Collapse in the International System, Yale University Press, 1995. Ces distinctions quoi qu'ayant cours ont été néanmoins bouleversées par la guerre avec les Soviétiques, la guerre civile et enfin l'arrivée des Taliban.

1 Pour ce récit et des commentaires, cette partie s'appuie sur D.B. Edwards, The Heroes of the Age, California, University of California Press, 1996.

8 Les Deux États, Pouvoir et société en Occident et en terre d'Islam, Paris, Fayard, 1986.

9 Voir entre autres, Peter Marsden, The Taliban, War, Religion and The New Order in Afghanistan, London, New York, Zed Books, 1998.

10 Le Monde Diplomatique, novembre 1996.

1 Voir notre article «L'idée de nation en Asie centrale» Transitions, vol. XXXVII, 1996-2, pp. 143-173.

12 Le soufisme a joué un rôle très important en Asie centrale, dans le pays tatar et le Caucase nord. Les ordres soufis représentaient la face populaire de l'islam, spécialement au XIIIe siècle. Ces ordres furent les garants de la sauvegarde de l'islam au temps de l'invasion monghole et au XIXe siècle face aux Russes. Voir l'œuvre d'A. Benningsen, entre autres, Le soufi et le commissaire, les confréries musulmanes en URSS, Paris, Seuil 1986.

13 Tendance islamique puritaine née au Nadjid, au XVIII e siècle.

14 Les conditions nécessaires pour acquérir le statut de République de l'Union seront énoncées par Staline en 1936. La république en question ne doit pas être entourée de tous côtés par le territoire de l'URSS, car les Républiques de l'union ayant le droit à la sécession, ce droit n'aurait pas de sens dans ces cas là. Ensuite la nationalité qui donne son nom à la république soviétique doit être majoritaire dans celle-ci. Enfin, la population d'une république doit dépasser le million, car en deça de ce nombre elle aurait du mal à protéger son indépendance.

15 Voir Pierre Lorrain, «La fureur du Caucase», Le Spectacle du Monde, octobre 1999.

16 Idem.

17 Alexê̂ Malashenko, «Du Daghestan à la Tchétchénie, escalade militaire et "péril islamique», Le Monde Diplomatique, octobre 1999. 